\title{
Real analytic Bernoulli geodesic flows on $\boldsymbol{S}^{2}$
}

\author{
KEITH BURNS AND MARLIES GERBER \\ Department of Mathematics, Indiana University, Bloomington, IN 47405, USA
}

(Received 29 January 1987 and revised 12 June 1987)

Abstract. We obtain a family of metrics on the two-dimensional sphere whose geodesic flow is ergodic and Bernoulli. This family includes real analytic metrics.

\section{Introduction}

We show that there is a real analytic metric on the two dimensional sphere $S^{2}$ whose geodesic flow is ergodic and Bernoulli. Metrics with Bernoulli (and therefore ergodic) geodesic flow that are $C^{1}$ but not $C^{2}$ were described by R. Osserman [O]. The simplest of them is obtained by attaching a hemisphere to each boundary geodesic of a pair of pants with hyperbolic metric (see, e.g. [FLP, Ch. 3]). His arguments relied heavily on the symmetry of the hemispheres. Burago has recently found a $C^{1}$ metric whose geodesic flow has the $K$ property [Bu]. V. Donnay [D1, D2, D3] has obtained $C^{\infty}$ metrics whose geodesic flows have almost everywhere nonzero Lyapunov exponents. In [D2] he outlines a proof that these examples have ergodic geodesic flows. Donnay's examples are similar to Osserman's. Instead of hemispheres, he attaches caps that are radially symmetric surfaces with positive curvature that decreases to zero on the boundary. The metric on the pair of pants is deformed so that the caps can attach smoothly along the boundary geodesics. Osserman's simple argument no longer applies, but Donnay showed that the Lyapunov exponents are almost everywhere nonzero by applying $M$. Wojtkowski's method of invariant cones $[\mathbf{W}]$.

We begin by studying $C^{\infty}$ examples similar to Donnay's. Our caps are radially symmetric disks bounded by closed geodesics in which the curvature increases with distance from the boundary. Following Donnay, we construct an invariant family of cones. The curvature condition on the caps simplifies the proof that the cones are invariant. We then show that the invariance property of the cones persists under suitable small perturbations. The class of allowable perturbations is large enough to include a real analytic metric. The family of invariant cones is continuous and hence the results of [BG] and [Ka] can be used to show that the geodesic flow is ergodic and Bernoulli. This approach seems simpler than that in [D2, D4].

We are grateful to A. Katok for suggesting the problem of finding real analytic metrics with ergodic geodesic flows. We also thank H. Bercovici, A. Katok, R. Spatzier and S. Zucker for helpful conversations. 
The second author is grateful to the Sonderforschungsbereich, Göttingen for support and warm hospitality during the time this paper was written.

\section{Description of examples and statement of results}

By a cap we will mean a closed two dimensional disk with nonnegative curvature such that the boundary circle is a geodesic. We say that a cap has monotone curvature if it is radially symmetric and its curvature is a nondecreasing function of distance from the boundary. Such a cap will be called nondegenerate if this function has positive derivative at the boundary and vanishes there.

THEOREM 1.1. Let $(S, g)$ be a compact $C^{\infty}$ Riemannian surface. Suppose that the set on which the curvature is nonnegative is a union of disjoint caps with monotone curvature $\mathscr{C}_{1}, \ldots, \mathscr{C}_{n}$. Then the geodesic flow $\varphi_{\mathrm{g}}^{\prime}$ on the unit tangent bundle $T^{1, g} S$ is ergodic and Bernoulli.

Donnay [D4] gives a different proof of ergodicity of the geodesic flow under essentially similar conditions. That proof and the argument in the present paper are based on the fundamental result that the Lyapunov exponents are almost everywhere nonzero [D1, D2, D3]. Once ergodicity has been proved, the Bernoulli property follows from a result of Katok [Ka].

It is clear from Proposition 2.1 that metrics with the properties required by Theorem 1.1 can be constructed on any compact $C^{\infty}$ surface as follows. Choose a finite set of points whose complement in the surface supports a hyperbolic metric with finite volume. Cut off the cusps of this hyperbolic surface along horocycles and deform the resulting surface with boundary so that the boundary circles are geodesics and the curvature is nonpositive and approaches 0 at the boundary. This can be done so that each boundary circle has an annular neighbourhood isometric to a surface of revolution in which the curvature at distance $r$ from the boundary is a monotone function of $r$ on $[0, \delta]$ for some $\delta>0$; moreover, we can arrange for this function to extend to a monotone $C^{\infty}$ function on $[-\delta, \delta]$. Now Proposition 2.1 allows us to smoothly attach a cap with monotone curvature along the boundary circle. The Gauss-Bonnet theorem imposes the restriction that there are at least three caps if the surface is $S^{2}$ and at least one cap if the surface is $T^{2}$. Note also that the surface must contain points of negative curvature, since it cannot be a disjoint union of caps.

The main result of this paper is

THEOREM 1.2. Suppose in addition that each cap $\mathscr{C}_{i}$ of $(S, g)$ is nondegenerate and has a neighbourhood in which the metric is radially symmetric about the centre of $\mathscr{C}_{i}$. Let $\tilde{\mathrm{g}}$ be a $C^{3}$ Riemannian metric that agrees with $\mathrm{g}$ to second order along each $\partial \mathscr{C}_{i}$. Fix a neighbourhood $\mathcal{N}_{i}$ of each $\partial \mathscr{C}_{i}$. If $\tilde{g}$ is close enough to $\mathrm{g}$ in the $C^{2}$ topology (throughout $S$ ) and $\tilde{g} \mid \mathcal{N}_{i}$ and $g \mid \mathcal{N}_{i}$ are close enough in the $C^{3}$ topology for each $i$, then the geodesic flow $\varphi_{\tilde{s}}^{\prime}$ on $T^{1, \tilde{s}} S$ is ergodic and Bernoulli with respect to its Liouville measure. 
In $\S 5$ we derive

COROLLARY 1.3. Every compact real analytic surface has a real analytic metric whose geodesic flow is ergodic and Bernoulli.

Theorems 1.1 and 1.2 are proved using the result of [BG], which is based on work of Pesin and Wojtkowski, and a result of Katok [Ka]. The result that we shall actually use is

THEOREM 1.4. Let $M$ be a compact three dimensional $C^{3}$ manifold, and let $\varphi^{\prime}$ be a $C^{2}$ flow that leaves invariant the measure defined by a $C^{2}$ Riemannian metric on $M$. Suppose that there is a $\varphi^{t}$-invariant distribution $P$ transverse to the flow. Let $\mathscr{K}$ be a continuous family of two dimensional cones such that $\mathscr{K}(x) \subseteq P(x)$ for all $x \in M$. Suppose that there is a connected open subset $\mathcal{U}$ of $M$ such that

(i) $\bigcup_{t \in \mathbf{R}} \varphi^{t} \mathcal{U}$ has full measure in $\boldsymbol{M}$;

(ii) $d \varphi^{t}(\mathscr{K}(u))$ is strictly contained in $\mathscr{K}\left(\varphi^{t} u\right)$ whenever $u \in \mathscr{U}, t>0$ and $\varphi^{t} u \in \mathscr{U}$. Then the flow $\varphi^{t}$ is ergodic. If in addition $\varphi^{t}$ is a contact flow and $P$ is the kernel of the contact form, then $\varphi^{\prime}$ is Bernoulli.

Proof. Ergodicity follows from [BG]; the Bernoulli property follows from a result in [Ka].

In our applications of this theorem, $M$ will be one of the unit tangent bundles $T^{1,8} S$ or $T^{1, \tilde{8}} S$ and $\varphi^{\prime}$ the corresponding geodesic flow. Recall that a Riemannian metric $\langle\cdot, \cdot\rangle$ on $S$ induces a Riemannian metric on TS:

$$
\left\langle\langle\xi, \eta\rangle=\left\langle\xi_{H}, \eta_{H}\right\rangle+\left\langle\xi_{V}, \eta_{V}\right\rangle\right.
$$

where $H$ and $V$ denote the horizontal and vertical components, respectively, see e.g. $[K 1, \S 3.2]$. We will identify $\xi \in T T S$ with $\left(\xi_{H}, \xi_{V}\right)$. The geodesic flow $\varphi^{t}$ on $T S$ is defined by the vector field $v \rightarrow(v, 0)$. The measure defined by $\langle\cdot \cdot, \cdot\rangle$ is invariant under the geodesic flow. Since the metric $\tilde{\mathrm{g}}$ in Theorem 1.2 is $C^{3}$, both the geodesic flow and the metric that it defines on $T S$ are $C^{2}$, and $T^{1, \tilde{g}} S$ is a $C^{3}$ submanifold of TS.

If $w \in T^{1} S$ set

$$
P(w)=\left\{\xi \in T_{w} T^{1} S:\left\langle\xi_{H}, w\right\rangle=0=\left\langle\xi_{V}, w\right\rangle\right\}
$$

and

$$
\mathscr{K}(w)=\left\{\xi \in P(w):\left\langle\xi_{H}, \xi_{V}\right\rangle \geq 0\right\} .
$$

Note that $P(w)$ is the orthogonal complement of the geodesic flow in the tangent space at $w$ of $T^{1} S$. It is well known that the distribution $P$ is invariant under the geodesic flow. Continuity of $\mathscr{K}$ is obvious. The 1 -form $\omega$ defined by

$$
\omega(\xi)=\left\langle\xi_{H}, u\right\rangle \quad \text { for } \xi \in T_{u} T^{1} S
$$

makes $\varphi^{\prime}$ a contact flow: $\omega$ is invariant under $\varphi^{\prime}$ and the 3 -form $\omega \wedge d \omega$ is everywhere nondegenerate and defines the same measure as the metric $\langle\langle\cdot, \cdot\rangle$. Before describing the proofs of Theorems 1.1 and 1.2, we reformulate property (ii) from Theorem 1.4 in terms of Jacobi fields. Recall that if $u$ is a unit vector and $\xi \in T_{u} T S$, then $Y(t)=\left(d g^{\prime} \xi\right)_{H}$ is a Jacobi field along the geodesic $\gamma_{u}$ determined by $u$ with covariant 
derivative $Y^{\prime}(t)=\left(d g^{t} \xi\right)_{v}$. Since $S$ is a surface, $Y(t)$ has the form

$$
Y(t)=y(t) N(t)+(a+b t) \dot{\gamma}_{u}(t),
$$

where $a$ and $b$ are constants, $N$ is a continuous field of unit normals to $\gamma_{u}$ and $y$ satisfies the scalar Jacobi equation

$$
y^{\prime \prime}(t)+K\left(\gamma_{u}(t)\right) y(t)=0,
$$

where $K(p)$ is the curvature at $p \in S$. (Conversely, a vector field $Y$ satisfying (1) and (2) arises from some $\xi \in T_{u} T S$.) It is well known that $\xi \in T_{u} T^{1} S$ if and only if $b=0$, and $\xi \in P(u)$ if and only if $a=b=0$. (It is clear from the latter fact that the distribution $P$ is invariant under the geodesic flow.)

The cone $\mathscr{K}(u)$ corresponds to the set of solutions of (2) with $y(0) y^{\prime}(0) \geq 0$. Property (ii) of the set $U$ in Theorem 1.4 is equivalent to

(ii') If $u \in \mathscr{U}, T>0, \varphi^{T} u \in \mathscr{U}$ and $y$ is a solution of (2) with $y(0) y^{\prime}(0) \geq 0$, then $y(T) y^{\prime}(T)>0$ unless $y(t) \equiv 0$.

Proof of Theorem 1.1. We apply Theorem 1.4 to $\varphi_{\mathrm{g}}^{\prime}$ on $T^{1, g} S$. We choose $\mathcal{U}$ to be the set of unit vectors whose footpoints lie outside the caps. Proposition 2.4 shows that $\mathcal{U}$ has property (i) required by Theorem 1.4. To show that $\mathcal{U}$ has property (ii), we verify (ii') above. Since $(S, g)$ has negative curvature outside the caps, (ii') follows easily from Lemma 1.5 below and Proposition 2.7, which describes what happens when a $\varphi^{\prime}$-orbit leaves and then reenters $\mathcal{U}$.

LEMMA 1.5. Let $y$ be a solution of (2). Suppose that $K\left(\gamma_{u}(t)\right)<0$ for $0<t<T$ and $y(t) \not \equiv 0$. If $y(0) y^{\prime}(0) \geq 0$, then $y(T) y^{\prime}(T)>0$.

Proof. $\left(y y^{\prime}\right)^{\prime}(t)=-K\left(\gamma_{u}(t)\right) y^{2}(t)+\left(y^{\prime}(t)\right)^{2}>0$ for $0<t<T$.

Proof of Theorem 1.2. We may assume that $\tilde{g}$ has positive curvature in $\bigcup$ Int $\mathscr{C}_{i}$ and negative curvature outside $\cup \mathscr{C}_{i}$. To see this, note that the nondegeneracy of the caps $\left(\mathscr{C}_{i}, g\right)$ means that if $\tilde{\mathrm{g}} \mid \mathcal{N}$ and $g \mid \mathcal{N}$ are close enough in the $C^{3}$ topology, there is a neighbourhood $\mathcal{N}_{0}$ of $\bigcup \partial \mathscr{C}_{i}$ such that the curvature of $\tilde{g}$ is positive on $\mathcal{N}_{0} \cap\left(\bigcup\right.$ Int $\left.\mathscr{C}_{i}\right)$ and negative on $\mathcal{N}_{0} \backslash\left(\bigcup \mathscr{C}_{i}\right)$. If $\tilde{g}$ and $g$ are close enough in the $C^{2}$ topology, the curvature of $\tilde{g}$ will also be positive on $\bigcup \mathscr{C}_{i} \backslash \mathcal{N}_{0}$ and negative outside $\mathcal{N}_{0} \cup\left(\bigcup \mathscr{C}_{i}\right)$.

We apply Theorem 1.4 to $\varphi_{\tilde{g}}^{t}$ on $T^{1, \tilde{z}} S$. For each cap $\mathscr{C}_{i}$, choose a closed disk $\mathscr{D}_{i}$ with $\mathscr{C}_{i} \subseteq$ Int $\mathscr{D}_{i}$ such that $\mathscr{D}_{1}, \ldots, \mathscr{D}_{n}$ are pairwise disjoint and $\left(\mathscr{D}_{i}, g\right)$ is radially symmetric about the centre of $\left(\mathscr{C}_{i}, g\right)$. Let $U$ be the set of vectors in $T^{1, \tilde{g}} S$ whose footpoints lie outside $\cup \mathscr{D}_{i}$. We verify that $\mathcal{U}$ satisfies the properties (i) and (ii') described above. Proposition 2.4 shows that the only geodesics of $(S, \tilde{g})$ that can stay in $\mathscr{C}_{i}$ for all time are parametrizations of $\partial \mathscr{C}_{i}$. Since the curvature of $\tilde{g}$ is negative in $\mathscr{D}_{i} \backslash \mathscr{C}_{i}$, dist $\tilde{g}_{\tilde{g}}\left(\mathscr{C}_{i}, \cdot\right)$ is a strictly convex function on $\mathscr{D}_{i} \backslash \mathscr{C}_{i}$ (see e.g. $\S 1 \mathrm{C}$ of [BGS]). Thus a geodesic that exits $\mathscr{C}_{i}$ leaves $\mathscr{D}_{i}$ after a finite time. This proves property (i). Since the curvature of $(S, \tilde{\boldsymbol{g}})$ is negative outside $\bigcup \mathscr{D}_{i}$, property (ii') follows from Proposition 4.10 and Lemma 1.5.

The main idea in both proofs is to show that the effect of the negative curvature on Jacobi fields is not destroyed when a geodesic passes through a $\mathscr{C}_{i}$. For Theorem 
1.1 , this is done by a direct study of the geometry of radially symmetric caps. The point in Theorem 1.2 is that the geometry of the caps $\mathscr{C}_{i}$ does not change significantly if $\tilde{g}$ is close enough to $g$.

\section{Geometry of caps}

We begin by constructing examples of caps with monotone curvature that vanishes at the boundary. The examples have constant positive curvature except in a neighbourhood of the boundary. The construction allows us to prescribe the circumference of the cap and the behaviour of the curvature near the boundary.

Proposition 2.1. Let $L>0$ and $k_{0}: \mathbb{R} \rightarrow \mathbb{R}$ a nondecreasing $C^{\infty}$ function with $k_{0}(0)=0$. Then there exist $R, \delta>0$ and a radially symmetric metric on a disk $\mathscr{D}$ with radius $R+\delta$ such that

(i) the disk $\mathscr{C}$ of radius $R$ about the centre of $\mathscr{D}$ is a cap with monotone curvature;

(ii) $\partial \mathscr{C}$ has length $L$;

(iii) if $s$ is the signed distance from $\partial \mathscr{C}$, chosen so that $s>0$ inside $\mathscr{C}$, then the curvature of $\mathscr{D}$ at distance $s$ from $\partial \mathscr{C}$ is $k_{0}(s),-\delta \leq s \leq \delta$.

The proof uses a standard comparison result for second order differential equations.

LEMMA 2.2. Suppose $K_{1}(t) \leq K_{2}(t)$ for $0 \leq t \leq T$ and $y_{i}(t), i=1,2$, is a solution for $0 \leq t \leq T$ of

$$
y_{i}^{\prime \prime}(t)+K_{i}(t) y_{i}(t)=0, \quad i=1,2 .
$$

If $y_{1}(0)=y_{2}(0), 0 \geq y_{1}^{\prime}(0) \geq y_{2}^{\prime}(0)$ and $y_{2}(t)>0$ for $0 \leq t<T$, then $y_{1}(t) \geq y_{2}(t)$ for $0 \leq t \leq T$.

Proof. Let $w(r)=\left(y_{1}^{\prime} y_{2}-y_{1} y_{2}^{\prime}\right)(r)$. Then $w(0) \geq 0$ and $w^{\prime}=\left(K_{2}-K_{1}\right) y_{1} y_{2}$. Thus $w(r) \geq 0$ if $y_{1}(s) y_{2}(s) \geq 0$ on $(0, r)$. The lemma follows since $\left(y_{2} / y_{1}\right)^{\prime}=-w / y_{1}^{2}$.

Proof of Proposition 2.1. Choose a continuous function $k:(0, \infty) \times \mathbb{R} \rightarrow \mathbb{R}$ such that for each $\eta>0$,

(I) $k(\eta, \cdot)$ is $C^{\infty}$, nondecreasing and has $k(\eta, 0)=0$;

(II) there is $\delta(\eta), 0<\delta(\eta)<\pi / 4 \eta$ such that $k(\eta, s)=k_{0}(s)$ for $|s| \leq \delta(\eta)$;

(III) $k(\eta, s)=\eta^{2}$ for all $s \geq \pi / 4 \eta$.

Let $l_{\eta}$ be the solution of

$$
l^{\prime \prime}(s)+k(\eta, s) l(s)=0
$$

with $l_{\eta}(0)=L$ and $l_{\eta}^{\prime}(0)=0$. Let $R(\eta)$ be the first positive value of $s$ with $l_{\eta}(s)=0$. Since $k(\eta, \cdot) \leq \eta^{2}$, it is clear from Lemma 2.2 that $R(\eta) \geq \pi / 2 \eta$. We shall show below that there is $\eta^{*}$ such that $l_{\eta^{*}}^{\prime}\left(R\left(\eta^{*}\right)\right)=-2 \pi$.

Choose $R=R\left(\eta^{*}\right)$ and $\delta=\delta\left(\eta^{*}\right)$. Let $\mathscr{D}$ be the disk of radius $R+\delta$ with the radially symmetric metric in which the curvature at distance $r$ from the centre $p_{0}$ is $\kappa(r)=k\left(\eta^{*}, R-r\right)$. To see that this metric is well defined, note firstly that $\kappa$ extends to a $C^{\infty}$ even function on $[-R-\delta, R+\delta]$ since $\kappa \equiv\left(\eta^{*}\right)^{2}$ on $[0, R / 2]$. Now observe that the circle of radius $r$ about $p_{0}$ in this metric should have length $\lambda(r)$ where $\lambda$ is the solution of the initial value problem

$$
\lambda^{\prime \prime}(r)+\kappa(r) \lambda(r)=0, \quad \lambda(0)=0, \quad \lambda^{\prime}(0)=2 \pi ;
$$


the metric will be well defined if $\lambda(r)>0$ for $0<r \leq R+\delta$. We see, however, that $\lambda(r)=l_{\eta^{*}}(R-r)$, which is nonzero for $0<r$. The circle about $p_{0}$ of radius $R$ has length $\lambda(R)=l_{\eta^{*}}(0)=L$. It is a geodesic since $\lambda^{\prime}(R)=-l_{\eta^{*}}^{\prime}(0)=0$, [see e.g. 3.5.22]. Claims (i), (ii), and (iii) now follow easily.

To show that we can choose $\eta^{*}$, it is enough, since $l_{\eta}^{\prime}(R(\eta))$ depends continuously on $\eta$, to show that

(a) $l_{\eta}^{\prime}(R(\eta))>-2 \pi$ if $\eta$ is sufficiently small and

(b) $l_{\eta}^{\prime}(R(\eta))<-2 \pi$ if $\eta$ is sufficiently large.

Note that $0<l_{\eta}(s) \leq L, l_{\eta}^{\prime}(s) \leq 0$, and $0 \leq k(\eta, s) \leq \eta^{2}$ for $0 \leq s<R(\eta)$. We saw above that $R(\eta) \geq \pi / 2 \eta$. Thus $l_{\eta}(\pi / 4 \eta)>0$ and $l_{\eta}^{\prime}(\pi / 4 \eta) \leq 0$. Since $k(\eta, s) \equiv \eta^{2}$ for $s \geq \pi / 4 \eta$, it follows from Lemma 2.2 that $R(\eta) \leq 3 \pi / 4 \eta$. Thus, by (3),

$$
l_{\eta}^{\prime}(R(\eta))=-\int_{0}^{R(\eta)} k(\eta, s) l_{\eta}(s) d s \geq-\eta^{2} L R(\eta) \geq-\frac{3 L \eta \pi}{4}>-2 \pi
$$

if $\eta$ is small. This proves (a). Since $l_{\eta}^{\prime \prime}>0$ on $[0, R(\eta)]$, we have

$$
l_{\eta}^{\prime}(R(\eta)) \leq\left(l_{\eta}(R(\eta))-l_{\eta}(0)\right) / R(\eta)=-L / R(\eta) \leq-4 L \eta / 3 \pi
$$

which is less than $-2 \pi$ for large $\eta$. This proves (b).

Now we study the behaviour of geodesics in an arbitrary cap.

LEMMA 2.3. Let $\gamma$ be a geodesic (parametrized by arc length) in a cap $\mathscr{C}$. Set $d(t)=\operatorname{dist}(\gamma(t), \partial \mathscr{C})$. Then $d$ is a concave function.

Proof. We show that for each $t$ there is a linear function $L_{t}(\tau)$ such that $d(t)=L_{t}(0)$ and $d(t+\tau) \leq L_{t}(\tau)$ for all $\tau$ sufficiently close to 0 . Choose a minimizing geodesic $\sigma$ from $\partial \mathscr{C}$ to $\gamma(t)$ and parametrize $\sigma$ so that $\sigma(0) \in \partial \mathscr{C}$ and $\sigma(d(t))=\gamma(t)$. We define

$$
L_{t}(\tau)=d(t)+\tau\langle\dot{\gamma}(t), \dot{\sigma}(d(t))\rangle .
$$

Let $V(s)$ be the vector field along $\sigma$ with $V(d(t))=\dot{\gamma}(t), V(0)$ tangent to $\partial \mathscr{C}$ and covariant derivative

$$
V^{\prime}(s)=\frac{1}{d(t)}\langle\dot{\gamma}(t), \dot{\sigma}(d(t))\rangle \dot{\sigma}(s) .
$$

Set $\alpha(s, \tau)=\gamma_{V(s)}(\tau)$. Then $(\partial \alpha / \partial s)(s, \cdot)$ is the Jacobi field along $\gamma_{V(s)}$ with initial value $\dot{\sigma}(s)$ and initial covariant derivative $V^{\prime}(s)$. Comparison with a Jacobi field in zero curvature using the Rauch theorem [CE, Theorem 1.28] shows that

$$
\left\|\frac{\partial \alpha}{\partial s}(s, \tau)\right\| \leq 1+\frac{\tau}{d(t)}\langle\dot{\gamma}(t), \dot{\sigma}(d(t))\rangle
$$

for any small enough $\tau$. The inequality $d(t+\tau) \leq L_{t}(\tau)$ follows, since

$$
d(t+\tau) \leq \int_{0}^{d(t)}\left\|\frac{\partial \alpha}{\partial s}(s, \tau)\right\| d s
$$

Proposition 2.4. Let $\gamma$ be a maximal geodesic in a cap $\mathscr{C}$. Then either $(i) \gamma$ is a closed geodesic, dist $(\gamma(t), \partial \mathscr{C})$ is constant and the curvature vanishes identically in the cylinder bounded by $\gamma$ and $\partial \mathscr{C}$; or (ii) $\gamma$ is a segment of finite length joining two points of $\partial \mathscr{C}$. Proof. If $d^{\prime}(t)<0$ for some $t$, then $\gamma$ hits $\partial \mathscr{C}$ after finite time. If $d^{\prime}(t) \geq 0$ for all $t$, then $\gamma$ is asymptotic to a closed geodesic $\gamma_{0}(s)$ with dist $\left(\gamma_{0}(s), \partial \mathscr{C}\right) \equiv \lim _{t \rightarrow \infty} h(t)$. 
The Gauss-Bonnet theorem shows that the curvature vanishes between $\partial \mathscr{C}$ and $\gamma_{0}$. It follows easily that $\gamma$ must be the closed geodesic $\gamma_{0}$.

In the rest of this section, we study a cap $\mathscr{C}$ whose curvature is monotone and vanishes on $\partial \mathscr{C}$. Let $\gamma$ be a maximal geodesic of $\mathscr{C}$ of type (ii). Parametrize $\gamma$ by arc length so that $\gamma\left( \pm T_{0}\right)$ are the points where $\gamma$ meets $\partial \mathscr{C}$. It is clear from the radial symmetry that $\gamma(0)$ is the unique point of $\gamma$ closest to the centre of $\mathscr{C}$. We study the scalar Jacobi equation along $\gamma$,

$$
y^{\prime \prime}(t)+\kappa(t) y(t)=0
$$

where $\kappa(t)$ is the curvature at $\gamma(t)$. Note that the function $\kappa$ is even on $\left[-T_{0}, T_{0}\right]$ and nonincreasing on $\left[0, T_{0}\right]$. Let $S$ and $C$ be the solutions of (4) with $C(0)=1, C^{\prime}(0)=0, S(0)=0$ and $S^{\prime}(0)=1$. Clearly $S$ is an odd and $C$ an even function on $\left[-T_{0}, T_{0}\right]$.

Lemma 2.5. $S^{\prime}\left(T_{0}\right)=S^{\prime}\left(-T_{0}\right)=0$ and $S(t) \neq 0$ unless $t=0$.

Proof. Introduce normal coordinates $(r, 0)$ about the centre $p_{0}$ of $\mathscr{C}$, so $r$ is distance from $p_{0}$ and $o \in S^{1}$. The restriction of the vector field $\partial / \partial o$ to $\gamma$ is a Jacobi field (corresponding to a variation obtained by rotating $\gamma$ about $p_{0}$ ). Its component orthogonal to $\gamma$ vanishes only at $\gamma(0)$. Thus this component is a multiple of $S(t)$ and $S(t) \neq 0$ unless $t=0$. Note that along the geodesic $\partial \mathscr{C}, \partial / \partial o$ and $\partial / \partial r$ are tangent and normal vector fields respectively with constant length. Thus along $\partial \mathscr{C}$, $0=\nabla_{\partial / \partial \sigma} \partial / \partial \sigma$ and $0=\nabla_{\partial / \partial o} \partial / \partial r=\nabla_{\partial / \partial r} \partial / \partial a$. Hence $\nabla_{v} \partial / \partial a=0$ for any vector $v$ with footpoint on $\partial \mathscr{C}$. In particular $\nabla_{\dot{\gamma}\left( \pm T_{0}\right)} \partial / \partial \circ=0$ and so $S^{\prime}\left( \pm T_{0}\right)=0$.

If $y$ is a non-trivial solution of (4), let $u=y^{\prime} / y$. Then $u$ satisfies the Riccati equation

$$
u^{\prime}(t)+u^{2}(t)+\kappa(t)=0
$$

Since $\kappa(t) \geq 0$, we have $u^{\prime}(t) \leq 0$. Note that $y(\tau)=0$ corresponds to a vertical asymptote at $t=\tau$ for the graph of $u$. Let $u_{S}=S^{\prime} / S$ and $u_{C}=C^{\prime} / C$. It follows from Lemma 2.5 that $u_{S}\left( \pm T_{0}\right)=0$ and the graph of $u_{S}$ is as shown in figure 1 .

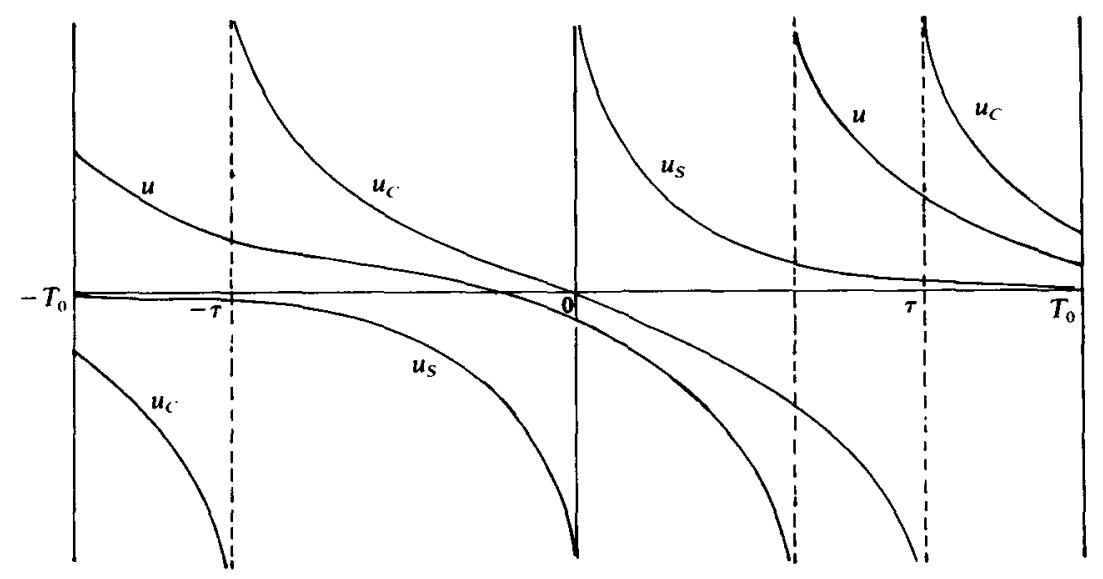

Figure 1 
LeMMA 2.6. There is $\tau \in\left(0, T_{0}\right]$ such that $\lim _{t \uparrow \tau} u_{C}(t)=-\infty$.

Proof. We prove that if $u_{C}(t)$ is finite for $0 \leq t<T_{0}$, then $u_{C}(t)<u_{S}\left(t-T_{0}\right)$ for $0<t<T_{0}$. This claim is obvious for any small enough $t$, since $u_{C}(0)=u_{S}\left(-T_{0}\right)=0$, $u_{S}^{\prime}\left(-T_{0}\right)=-\kappa\left(-T_{0}\right)=0$ and $u_{C}^{\prime}(0)=-\kappa(0)<0$. If our claim is false, there is $\tau_{1}<T_{0}$ such that $u_{C}(t)<u_{S}\left(t-T_{0}\right) \leq 0$ for $0<t<\tau_{1}$ and $u_{C}\left(\tau_{1}\right)=u_{S}\left(\tau_{1}-T_{0}\right)$. Since $\kappa$ is even on $\left[-T_{0}, T_{0}\right]$ and nonincreasing on $\left[0, T_{0}\right]$,

$$
\int_{0}^{\tau_{1}} \kappa(t) d t \geq \int_{0}^{\tau_{1}} \kappa\left(t-T_{0}\right) d t
$$

Thus we obtain the contradiction

$$
\begin{aligned}
u_{C}\left(\tau_{1}\right) & =-\int_{0}^{\tau_{1}}\left\{\kappa(t)+u_{C}^{2}(t)\right\} d t \\
& <-\int_{0}^{\tau_{1}}\left\{\kappa(t)+u_{S}^{2}\left(t-T_{0}\right)\right\} d t \\
& \leq-\int_{0}^{\tau_{1}}\left\{\kappa\left(t-T_{0}\right)+u_{S}^{2}\left(t-T_{0}\right)\right\} d t \\
& =u_{S}\left(\tau_{1}-T_{0}\right) .
\end{aligned}
$$

Since $u_{S}$ and $u_{C}$ are solutions of (5), their graphs cannot cross. It follows from Lemma 2.6 that the graph of $u_{C}$ is as in figure 1 .

Consider a solution $y$ of (4) that has $y\left(-T_{0}\right) y^{\prime}\left(-T_{0}\right) \geq 0$. Since $u=y^{\prime} / y$ satisfies (5), the graph of $u$ must lie between the graphs of $u_{S}$ and $u_{C}$, as shown in figure 1. It follows that $y\left(T_{0}\right) y^{\prime}\left(T_{0}\right) \geq 0$. We have proved

Proposition 2.7. Let $\gamma$ be a geodesic that enters $\mathscr{C}$ at time $-T_{0}$ and leaves at time $T_{0}$. Let $y$ be a solution of the scalar Jacobi equation along $\gamma$ such that $y\left(-T_{0}\right) y^{\prime}\left(-T_{0}\right) \geq 0$. Then $y\left(T_{0}\right) y^{\prime}\left(T_{0}\right) \geq 0$.

\section{Geometry of nondegenerate caps}

Our goal in the next two sections is to control the behaviour of Jacobi fields of the perturbed surface $(S, \tilde{g})$ in Theorem 1.2 as geodesics pass through the caps $\mathscr{C}_{i}$. Eventually we shall obtain in Proposition 4.10 an analogue of Proposition 2.7. The idea behind its proof is that geodesics and Jacobi fields for $(S, \tilde{g})$ should closely follow those of $(S, g)$. The main difficulty is that the time needed for a geodesic $\gamma$ of $(S, g)$ to pass through one of the caps $\mathscr{C}_{i}$ is not uniformly bounded. Since the curvature vanishes at the boundary of $\mathscr{C}_{i}, \gamma$ will stay in $\mathscr{C}_{i}$ for an arbitrarily long time if the angle $\alpha$ at which it crosses the boundary is small enough. We deal with this problem in $\S 4$ by rescaling $g$ and $\tilde{g}$ near the boundary of the cap by the factor $\alpha^{2 / 3}$. In this section we estimate the time taken by $\gamma$ to cross the cap $\mathscr{C}_{i}$ in terms of $\alpha$; see Lemma 3.4. The dependence on $\alpha$ of this estimate will disappear after the rescaling.

A second difficulty is that the solution $u_{S}$ of the Riccati equation vanishes both when $\gamma$ is entering and when $\gamma$ is leaving the cap. It is clear from this that the invariance property proved in Proposition 2.7 can be destroyed by arbitrarily small 
perturbations. This problem is overcome in Lemma 3.6 by following $\gamma$ for some time before and after its passage through the cap. The negative curvature outside the cap makes $u_{s}$ increase before it enters and after it leaves the cap. The extra time during which we follow $\gamma$ depends on the angle $\alpha$; this dependence will also disappear after the rescaling of $g$ by $\alpha^{2 / 3}$.

We emphasize that the results of this section are for a nondegenerate cap. Similar estimates should hold whenever the curvature changes sign at the boundary of the cap and some derivative of the curvature perpendicular to the boundary is nonzero.

Let $\mathscr{C}$ be a nondegenerate cap with monotone curvature contained in a larger disk $\mathscr{D}$ whose metric is radially symmetric about the centre of $\mathscr{C}$ and whose curvature is negative outside $\mathscr{C}$. Let $s$ be the signed distance from $\partial \mathscr{C}$, chosen so that $s<0$ in $\mathscr{D} \backslash \mathscr{C}$. Let $l(s)$ be the length of the circle at distance $s$ from $\partial \mathscr{C}$ and $k(s)$ the curvature at points on this circle. We have

$$
l^{\prime \prime}(s)+k(s) l(s)=0 .
$$

Note that $k(s)$ is nondecreasing and $k(0)=0$. The nondegeneracy of the cap means that $k^{\prime}(0)>0$. Also $l^{\prime}(0)=0$, since $\partial \mathscr{C}$ is a geodesic [KI, 3.5.22]. It follows that

$$
\begin{array}{lll}
l^{\prime}(s) \leq 0 & \text { and } \quad l^{\prime \prime}(s) \leq 0, & \text { if } s \geq 0 ; \\
l^{\prime}(s) \leq 0 & \text { and } \quad l^{\prime \prime}(s) \geq 0, & \text { if } s \leq 0 .
\end{array}
$$

Let $\gamma$ be a geodesic in $\mathscr{D}$ that joins two points of $\partial \mathscr{C}$. Since the curvature is negative in $\mathscr{D} \backslash \mathscr{C}, \gamma$ does not reenter $\mathscr{C}$ after it leaves. As in $\S 2$ we parametrize $\gamma$ so that $\gamma\left( \pm T_{0}\right)$ are the points where $\gamma$ crosses $\partial \mathscr{C}$. Set $z(t)=s(\gamma(t))$ and let $\Psi(t) \in[-\pi / 2, \pi / 2]$ be the angle that $\gamma$ makes with the circle on which $s$ is constant. The sign of $\Psi(t)$ is chosen so that $\Psi\left(-T_{0}\right) \geq 0$ and

$$
z^{\prime}(t)=\sin \Psi(t)
$$

The radial symmetry implies the constancy of the Clairaut integral

$$
l(z(t)) \cos \Psi(t) \equiv l(z(0))
$$

[KI, 3.5.23].

LemMA 3.1. $z^{\prime \prime}(t) \leq 0$ unless $\gamma(t)$ is the centre of $\mathscr{C}$.

Proof. We see from (8) and (9) that

$$
z^{\prime \prime}(t)=\frac{l^{\prime}(z(t))}{l(z(t))} \cos ^{2} \Psi(t) .
$$

The lemma follows from this and $(7 \mathrm{a}, \mathrm{b})$.

LEMMA 3.2. $z^{\prime \prime \prime}(t) \leq 0$ for $-T_{0}<t<0$.

Remark. Lemma 3.2 and its proof apply to any radially symmetric cap.

Proof. By (10),

$$
z^{\prime \prime}(t)=\frac{l^{\prime}(z(t))}{l^{3}(z(t))} l^{2}(z(t)) \cos ^{2} \Psi(t)
$$

Observe that $l^{2}(z(t)) \cos ^{2} \Psi(t)$ is constant, since it is the square of the Clairaut integral. Since $z(t)$ is increasing for $-T_{0} \leq t<0$ and $l^{\prime}\left(z\left(-T_{0}\right)\right)=l^{\prime}(0)=0$, it follows 
from (7a) that, for $-T_{0} \leq t<0, l^{\prime}(z(t))$ is nonpositive and nonincreasing while $l^{3}(z(t))$ is positive and nonincreasing.

Set $z_{\max }=z(0)$ and let $\alpha=\Psi(0)>0$ be the angle at which $\gamma$ crosses $\partial \mathscr{C}$. If $\alpha$ is small enough so that $\operatorname{dist}(\partial \mathscr{C}, \partial \mathscr{D}) \geq \alpha^{2 / 3}$, we define $T_{1}>0$ by $z\left(T_{1}\right)=-\alpha^{2 / 3}$. The radial symmetry of $\mathscr{D}$ means that $z_{\max }, T_{0}$ and $T_{1}$ are determined by $\alpha$.

LEMMA 3.3. There is a constant $C_{3}>1$ such that $C_{3}^{-1} \alpha^{2 / 3} \leq z_{\max }(\alpha) \leq C_{3} \alpha^{2 / 3}$ for any small enough $\alpha$.

Proof. It follows from (9) that $l\left(z_{\max }\right)=l(0) \cos \alpha=l(0)-\frac{1}{2} l(0) \alpha^{2}+o\left(\alpha^{3}\right)$ as $\alpha \rightarrow 0$. Since $l^{\prime}(0)=0$, and $l^{\prime \prime}(0)=-k(0) l(0)=0$,

$$
l(s)=l(0)-\frac{1}{6} l^{\prime \prime \prime}(0) s^{3}+o\left(s^{3}\right)
$$

as $s \rightarrow 0$. The lemma follows, since nondegeneracy of the cap implies $l^{\prime \prime \prime}(0)=$ $-k^{\prime}(0) l(0)<0$.

LEMMA 3.4. There is a constant $C_{4}>1$ such that for any small enough $\alpha$,

$$
\begin{gathered}
C_{4}^{-1} \alpha^{-1 / 3}<T_{0}(\alpha)<C_{4} \alpha^{-1 / 3} ; \\
C_{4}^{-1} \alpha^{-1 / 3}<T_{1}(\alpha)-T_{0}(\alpha)<C_{4} \alpha^{-1 / 3} .
\end{gathered}
$$

Proof. (a) Since $z^{\prime \prime} \leq 0$ on $\left(-T_{0}, 0\right), z^{\prime}(t) \leq z^{\prime}\left(-T_{0}\right)=\sin \alpha$ for $-T_{0} \leq t \leq 0$. Thus $z_{\max } \leq T_{0} \sin \alpha$ and the lower bound follows from Lemma 3.3. Lemma 3.2 implies that for $\frac{1}{2} T_{0} \leq t \leq T_{0}$

$$
\begin{aligned}
z^{\prime}(-t) & \geq\left(1-\frac{t}{T_{0}}\right) z^{\prime}(0)+\frac{t}{T_{0}} z^{\prime}\left(-T_{0}\right) \\
& =\frac{t}{T_{0}} z^{\prime}\left(-T_{0}\right) \geq \frac{1}{2} z^{\prime}\left(-T_{0}\right)=\frac{1}{2} \sin \alpha .
\end{aligned}
$$

Thus $z_{\max } \geq z\left(-\frac{1}{2} T_{0}\right) \geq-\frac{1}{4} T_{0} \sin \alpha$. The upper bound follows from Lemma 3.3.

(b) By Lemma 3.1,

$$
\sin \Psi\left(-T_{1}\right)=z^{\prime}\left(-T_{1}\right) \geq z^{\prime}(t) \geq z^{\prime}\left(-T_{0}\right)=\sin \alpha .
$$

Since $z\left(T_{0}\right)-z\left(T_{1}\right)=\alpha^{2 / 3}$, the upper bound on $T_{1}-T_{0}$ is clear. The lower bound will also follow if

$$
\sin \Psi\left(-T_{1}\right)=O(\alpha)
$$

We see from (9) that

$$
l\left(-\alpha^{2 / 3}\right) \cos \Psi\left(-T_{1}\right)=l\left(z_{\text {max }}\right) .
$$

We have $l^{\prime}(0)=l^{\prime \prime}(0)=0, l^{\prime \prime \prime}(0)<0$. Also $z_{\max }=O\left(\alpha^{2 / 3}\right)$ by Lemma 3.3. Thus there is a constant $B>0$ such that

$$
l\left(z_{\max }\right) \geq l(0)\left(1-B \alpha^{2}\right)
$$

and

$$
l\left(-\alpha^{2 / 3}\right) \leq l(0)\left(1+B \alpha^{2}\right) .
$$

Hence $\cos \Psi\left(-T_{1}\right) \geq 1-3 B \alpha^{2}$, which proves (11).

We now consider the Riccati equation (5) along $\gamma$ for $-T_{1} \leq t \leq T_{1}$. Define $u_{S}$ and $u_{C}$ as in $\S 2$. We saw in $\S 2$ that $0=u_{S}\left(T_{0}\right)<u_{C}\left(T_{0}\right) \leq \infty$. Since $K(\gamma(t)) \leq 0$ for 
$T_{0} \leq t \leq T_{1}$, it follows that

$$
0 \leq u_{S}(t)<u_{C}(t)<\infty \text { for } T_{0}<t \leq T_{1} .
$$

Lemma 3.5. There is a constant $C_{5}>1$ such that, for any small enough $\alpha$,

$$
C_{5}^{-1} \alpha^{1 / 3} \leq u_{S}\left(T_{1}(\alpha)\right)<u_{C}\left(T_{1}(\alpha)\right) \leq C_{5} \alpha^{1 / 3} \text {. }
$$

Proof. Let $u_{0}$ be the solution of (5) with $\lim _{t \downarrow T_{0}} u(t)=\infty$. Then $u_{C}(t) \leq u_{0}(t)$ for $T_{0}<t \leq T_{1}$. For such $t$ we have $0>z(t) \geq-\alpha^{2 / 3}$. Since the curvature is a smooth function on $\mathscr{D}$ and vanishes on $\partial \mathscr{C}$, there is a constant $B>0$ such that, for any small enough $\alpha, K(\gamma(t)) \geq-B^{2} \alpha^{2 / 3}$ for $T_{0} \leq t \leq T_{1}$. It follows that $u_{0}(t) \leq u_{1}(t)$ for $T_{0}<t \leqslant T_{1}$, where $u_{1}$ is the solution of the initial value problem

$$
u_{1}^{\prime}(t)+u_{1}^{2}(t)-B^{2} \alpha^{2 / 3}=0, \quad \lim _{t \downarrow T_{0}} u_{1}(t)=\infty .
$$

Hence $u_{C}(t) \leq B \alpha^{1 / 3} \operatorname{coth}\left(B \alpha^{1 / 3}\left(t-T_{0}\right)\right)$ for $T_{0}<t \leq T_{1}$. The desired upper bound on $u_{C}\left(T_{1}\right)$ follows, since $\alpha^{1 / 3}\left(T_{1}-T_{0}\right)$ is uniformly bounded from below, by Lemma 3.4(b).

If $t \geq T_{0}$, then $z^{\prime}(t) \leq z^{\prime}\left(T_{0}\right)=-\sin \alpha$, by Lemma 3.1. Since the cap $\mathscr{C}$ is nondegenerate, $k^{\prime}(0)>0$. Thus there is $A>0$ such that, for any small enough $\alpha$, we have $k^{\prime}(z(t)) z^{\prime}(t) \leq-2 A \sin \alpha \leq-A \alpha$ for $T_{0} \leq t \leq T_{1}$. Since $k\left(z\left(T_{0}\right)\right)=0$ we obtain $k(z(t)) \leq-A \alpha t$. It follows from Lemma 3.4(b) that there is a constant $C>0$ such that for all small enough $\alpha$ we have $K(\gamma(t)) \leq-C^{2} \alpha^{2 / 3}$ for $T_{1 / 2} \equiv \frac{1}{2}\left(T_{0}+T_{1}\right) \leq t \leq T_{1}$. Thus $u_{S}(t) \geq u_{2}(t)$ for $T_{1 / 2} \leq t \leq T_{1}$, where $u_{2}(t)$ is the solution of the initial value problem

Hence

$$
u_{2}^{\prime}(t)+u_{2}^{2}(t)-C^{2} \alpha^{2 / 3}=0, u_{2}\left(T_{1 / 2}\right)=0
$$

$$
u_{S}(t) \geq C \alpha^{1 / 3} \tanh \left(C \alpha^{1 / 3}\left(t-T_{1 / 2}\right)\right)
$$

for $T_{1 / 2} \leq t \leq T_{1}$. The lower bound on $u_{S}\left(T_{1}\right)$ follows since $\alpha^{1 / 3} T_{1 / 2}$ is uniformly bounded from below by Lemma 3.4(b).

\section{Perturbation of a nondegenerate cap}

As in $\S 3$, let $\mathscr{C}$ be a nondegenerate cap with monotone curvature contained in the interior of a disk $\mathscr{D}$ that is radially symmetric about the centre of $\mathscr{C}$ and has negative curvature outside $\mathscr{C}$. Denote by $g$ the metric on $\mathscr{D}$. Let $\tilde{\boldsymbol{g}}$ be another metric on $\mathscr{D}$ that agrees with $g$ to second order on $\partial \mathscr{C}$. Let $\mathcal{N}$ be a neighbourhood of $\partial \mathscr{C}$. We compare geodesics for $g$ and $\tilde{g}$ under the assumptions that $g \mid \mathcal{N}$ and $\tilde{g} \mid \mathcal{N}$ are $C^{3}$-close and that $g$ and $\tilde{g}$ are $C^{2}$ close. Let $\gamma$ be a geodesic of $(\mathscr{D}, g)$ that crosses $\partial \mathscr{C}$ at angle $\alpha$. Parametrize $\gamma$ as in $\S 3$, so that $\gamma\left(-T_{0}(\alpha)\right)$ and $\gamma\left(T_{0}(\alpha)\right)$ are the points where $\gamma$ enters and leaves $\mathscr{C}$ respectively. Let $\tilde{\gamma}$ be the $\tilde{\mathbf{g}}$-geodesic with $\dot{\gamma}\left(-T_{0}(\alpha)\right)=\dot{\gamma}\left(-T_{0}(\alpha)\right)$.

Mostly we study geodesics that make a small angle with $\partial \mathscr{C}$. Choose $\alpha_{0}$ so small that the estimates of Lemmas 3.3, 3.4 and 3.5 apply for any $\alpha \leq \alpha_{0}$. Set

$$
\mathcal{N}_{\alpha}=\left\{p \in \mathscr{D}: \operatorname{dist}_{\mathrm{g}}(p, \partial \mathscr{C}) \leq\left(C_{3}+1\right) \alpha^{2 / 3}\right\},
$$

where $C_{3}$ is the constant defined in Lemma 3.3. We assume that, for $\alpha \leq \alpha_{0}$, $\mathcal{N}_{\alpha} \subseteq \mathcal{N}, \mathcal{N}_{\alpha} \cap \partial \mathscr{D}=\varnothing$ and $\mathcal{N}_{\alpha}$ does not contain the centre of $(\mathscr{C}, g)$. 
The comparison between $\gamma$ and $\tilde{\gamma}$ is simplified if we rescale the metrics $g$ and $\tilde{g}$ by a factor of $\alpha^{2 / 3}$ to obtain the metrics $h_{\alpha}$ and $\tilde{h}_{\alpha}$ respectively. We have the following estimates for curvatures $K_{h_{\alpha}}$ and $K_{\tilde{h}_{\alpha}}$ of $h_{\alpha}$ and $\tilde{h}_{\alpha}$ on $\mathcal{N}_{\alpha}$.

LEMMA 4.1. (a) There is a constant $C>0$ such that $\left|K_{h_{\alpha}}(p)\right| \leq C$ whenever $\alpha \leq \alpha_{0}$ and $p \in \mathcal{N}_{\alpha}$.

(b) There is a constant $L>0$ such that $\left|K_{h_{\alpha}}(p)-K_{h_{\alpha}}(q)\right| \leq L \alpha^{-1} \operatorname{dist}_{h_{\alpha}}(p, q)$ whenever $\alpha \leq \alpha_{0}$ and $p, q \in \mathcal{N}_{\alpha}$.

Proof. Since the curvature $K_{\mathrm{g}}$ of $\mathrm{g}$ is a smooth function on $\mathscr{D}$, there is a constant $L>0$ such that $\left|K_{\mathrm{g}}(p)-K_{\mathrm{g}}(q)\right| \leq L \operatorname{dist}_{\mathrm{g}}(p, q)$ for any $p, q \in \mathcal{N}$. This implies (b), since $K_{h_{\alpha}}=\alpha^{-2 / 3} K_{\mathrm{g}}$ and dist ${ }_{h_{\alpha}}=\alpha^{1 / 3}$ dist $_{\mathrm{g}}$. Since $K_{\mathrm{g}}$ vanishes on $\partial \mathscr{C}$,

on $\mathcal{N}_{\alpha}$, which proves (a).

$$
\left|K_{g}(p)\right| \leq L\left(C_{3}+1\right) \alpha^{2 / 3}
$$

LEMMA 4.2. Let $\varepsilon>0$. If $g \mid \mathcal{N}$ and $\tilde{g} \mid \mathcal{N}$ are close enough in the $C^{3}$ topology, then

$$
\left|K_{h_{\alpha}}(p)-K_{\tilde{h}_{\alpha}}(p)\right| \leq \varepsilon
$$

whenever $\alpha \leq \alpha_{0}$ and $p \in \mathcal{N}_{\alpha}$.

Proof. Recall that curvature is determined by the second derivative of a metric and that $\boldsymbol{g}$ and $\tilde{g}$ agree to second order on $\partial \mathscr{C}$. Hence, given $\varepsilon^{\prime}>0$, we will have

$$
\left|K_{g}(p)-K_{\tilde{g}}(p)\right| \leq \varepsilon^{\prime} \operatorname{dist}_{g}(p, \partial \mathscr{C}) \leq \varepsilon^{\prime}\left(C_{3}+1\right) \alpha^{2 / 3}, \quad p \in \mathcal{N},
$$

provided $g \mid \mathcal{N}$ and $\tilde{g} \mid \mathcal{N}$ are sufficiently $C^{3}$-close.

Let $\beta(t)=\gamma\left(\alpha^{-1 / 3} t\right)$ and $\tilde{\beta}(t)=\tilde{\gamma}\left(\alpha^{-1 / 3} t\right)$. Then $\beta$ and $\tilde{\beta}$ are unit speed geodesics for $h_{\alpha}$ and $\tilde{h}_{\alpha}$ respectively. Set $t_{0}(\alpha)=\alpha^{1 / 3} T_{0}(\alpha)$ and $t_{1}(\alpha)=\alpha^{1 / 3} T_{1}(\alpha)$. Note that

$$
\beta(t) \in \mathcal{N}_{\alpha} \text { and } \operatorname{dist}_{h_{\alpha}}(\beta(t), \partial \mathcal{N}) \geq \alpha,
$$

for $-t_{1}(\alpha) \leq t \leq t_{1}(\alpha)$. Also

$$
C_{4}^{-1} \leq t_{0}(\alpha) \leq C_{4} \quad \text { and } \quad C_{4}^{-1} \leq t_{1}(\alpha)-t_{0}(\alpha) \leq C_{4},
$$

for all $\alpha \leq \alpha_{0}$, where $C_{4}$ is the constant defined in Lemma 3.4.

Proposition 4.3. Suppose $0<\varepsilon<1$. If $\mathrm{g} \mid \mathcal{N}$ and $\tilde{\mathrm{g}} \mid \mathcal{N}$ are close enough in the $C^{3}$ topology, then $\operatorname{dist}_{h_{\alpha}}(\beta(t), \tilde{\beta}(t)) \leq \varepsilon \alpha$ whenever $\alpha \leq \alpha_{0}$ and $-t_{1}(\alpha) \leq t \leq t_{1}(\alpha)$.

The proof uses a well known lemma from the theory of ordinary differential equations.

LEMMA 4.4. Let $X$ and $\tilde{X}$ be $C^{1}$ vector fields on a compact Riemannian manifold $M$ with boundary. Let $\varphi^{t}$ and $\psi^{t}$ be the flows defined by $X$ and $\tilde{X}$ respectively. Suppose that there are constants $A, B>0$ such that for any $m \in \mathcal{M}$ and $\xi \in T M$

(i) $\|X(m)-\tilde{X}(m)\| \leq A$;

(ii) $d /\left.d t\left(\left\|d \varphi^{\prime} \xi\right\|\right)\right|_{r=0} \leq B\|\xi\|$.

Let $m_{0} \in \mathcal{M} \backslash \partial \mathcal{M}$. Suppose that, for each $t \in(0, T), \varphi^{\prime} m_{0}, \psi^{t} m_{0} \in \mathcal{M} \backslash \partial \mathcal{M}$ and there is a minimal geodesic from $\varphi^{\prime} m_{0}$ to $\psi^{t} m_{0}$ that does not contain any point of $\partial \mathcal{M}$. Then

for $0 \leq t \leq T$.

$$
\operatorname{dist}\left(\varphi^{t} m_{0}, \psi^{\prime} m_{0}\right) \leq \frac{A}{B}\left(e^{B t}-1\right)
$$


Remark. The conditions on $\varphi^{\prime} m_{0}$ and $\psi^{t} m_{0}$ will follow if

$$
\operatorname{dist}\left(\varphi^{t} m_{0}, \partial \mathcal{M}\right)>\frac{A}{B}\left(e^{B T}-1\right) \quad \text { for } 0 \leq t \leq T \text {. }
$$

Proof. Let $l(t)=\operatorname{dist}\left(\varphi^{t} m_{0}, \psi^{t} m_{0}\right)$. For a given $t$, set $L_{1}(s)=\operatorname{dist}\left(\varphi^{s+t} m_{0}, \varphi^{s} \psi^{t} m_{0}\right)$ and $L_{2}(s)=\operatorname{dist}\left(\varphi^{s} \psi^{t} m_{0}, \psi^{s+t} m_{0}\right)$. Then

$$
l^{\prime}(t) \leq L_{1}^{\prime}(0)+L_{2}^{\prime}(0) \leq B l(t)+A,
$$

from which the lemma is clear. To see that $L_{1}^{\prime}(0) \leq B l(t)$, choose a minimal geodesic $\sigma$ with $\sigma(0)=\psi^{\prime} m_{0}$ and $\sigma(l(t))=\varphi^{t} m_{0}$. Then

$$
\begin{aligned}
L_{1}^{\prime}(0) & \leq\left.\frac{d}{d s}\left\{\int_{0}^{l(t)}\left\|d \varphi^{s} \dot{\sigma}(\tau)\right\| d \tau\right\}\right|_{s=0} \\
& \leq B \int_{0}^{l(t)}\|\dot{\sigma}(\tau)\| d \tau=B l(t) .
\end{aligned}
$$

We have $L_{2}^{\prime}(s) \leq A$ since $\varphi^{\prime} m_{0} \notin \partial \mathcal{M}$.

To prove Proposition 4.3, we will apply this lemma with

$$
M=M_{\alpha}=\left\{v \in T \mathcal{N}_{\alpha}:\|v\|_{h_{\alpha}} \leq 2\right\}=\left\{v \in T \mathcal{N}_{\alpha}:\|v\|_{g} \leq 2 \alpha^{-1 / 3}\right\} .
$$

We will use on $\mathcal{M}_{\alpha}$ the Riemannian metric $H_{\alpha}$ determined by $h_{\alpha}$ in the manner explained in $\S 1$. The vector fields $X$ and $\tilde{X}$ will be the infinitesimal generators of the geodesic flows for $h_{\alpha}$ and $\tilde{h}_{\alpha}$ respectively. The appropriate choices of $A$ and $B$ will be clear from the next two lemmas.

LeMmA 4.5. Let $\varepsilon>0$. If $\tilde{g}$ is sufficiently close to $g$ in the $C^{3}$ topology on $\mathcal{N}$, then $\|X(v)-\tilde{X}(v)\|_{H_{\alpha}} \leq \varepsilon \alpha$ for all $v \in M_{\alpha}$.

Proof. Let $x^{1}, x^{2}$ be Fermi coordinates for the geodesic $\partial \mathscr{C}$ with respect to the original metric $g$, so $x^{1}$ measures $g$-distance along $\partial \mathscr{C}$ and $x^{2}$ is the (signed) $g$-distance from $\partial \mathscr{C}$. Observe that the geodesic flow for $g$ (respectively $\tilde{g}$ ) on $T \mathcal{N}$ is exactly the same flow as the geodesic flow for $h_{\alpha}$ (respectively $\tilde{h}_{\alpha}$ ). Thus, in our coordinates,

$$
X\left(x^{1}, x^{2}, v^{1}, v^{2}\right)=\left(v^{1}, v^{2},-\Gamma_{i j}^{1}\left(x^{1}, x^{2}\right) v^{i} v^{j},-\Gamma_{i j}^{2}\left(x^{1}, x^{2}\right) v^{i} v^{j}\right),
$$

where

$$
\Gamma_{i j}^{k}=\frac{1}{2} g^{k l}\left\{\frac{\partial g_{i l}}{\partial x^{j}}+\frac{\partial g_{j l}}{\partial x^{i}}-\frac{\partial g_{i j}}{\partial x^{l}}\right\} .
$$

There are analogous formulas for $\tilde{X}$ and $\tilde{\Gamma}_{i j}^{k}$. The metrics $g$ and $\tilde{g}$ agree to second order on $\partial \mathscr{C}$. Thus if $g \mid \mathcal{N}$ and $\tilde{g} \mid \mathcal{N}$ are sufficiently close in the $C^{3}$ topology, we can assume that, for any given $\delta>0$,

$$
\left|\tilde{\Gamma}_{i j}^{k}-\Gamma_{i j}^{k}\right| \leq \delta\left(x^{2}\right)^{2}
$$

throughout $\mathcal{N}$. Let $\|\xi\|_{0}$ denote the length of $\xi \in T T \mathcal{N}$ in the Riemannian metric induced on $T \mathcal{N}$ by $g$. There is a constant $C>0$ such that, for $v=\left(x^{1}, x^{2}, v^{1}, v^{2}\right) \in T \mathcal{N}$,

$$
\|X(v)-\tilde{X}(v)\|_{0} \leq C \max _{1 \leq i, j, k \leq 2}\left|\tilde{\Gamma}_{i j}^{k}\left(x^{1}, x^{2}\right)-\Gamma_{i j}^{k}\left(x^{1}, x^{2}\right)\right| \max _{1 \leq i, j \leq 2}\left|v^{i} v^{j}\right| .
$$

It is clear from the definition of $\mathcal{N}_{\alpha}$ that, if we choose $\delta$ in (15) small enough, we 
will have

$$
\|X(v)-\tilde{X}(v)\|_{0} \leq \varepsilon \alpha^{2 / 3}
$$

for all $v \in \mathcal{M}_{\alpha}$. The lemma follows, since $\|\cdot\|_{H_{\alpha}}=\alpha^{1 / 3}\|\cdot\|_{0}$.

LEMMA 4.6. Let $S$ be a complete Riemannian surface whose curvature satisfies $|K(p)| \leq K_{0}$ for all $p \in S$. Let $\xi \in T_{v} T S$. The derivative of the geodesic flow $\varphi^{t}$ satisfies

$$
\left.\frac{d}{d t}\left(\left\|d \varphi^{t}(v) \xi\right\|\right)\right|_{t=0} \leq \frac{1}{2}\left\{1+K_{0}\|v\|^{2}\right\}\|\xi\| .
$$

Remark. This is the same result as Lemma 5.1 of [BBB]. Theorem 2 of [M] contains a related estimate.

Proof. Let $Y$ be the Jacobi field along $\gamma_{v}(t)$ for which $Y(t)$ and $Y^{\prime}(t)$ are the horizontal and vertical components of $d \varphi^{\prime}(v) \xi$. Then

$$
\left\|d \varphi^{\prime}(v) \xi\right\|^{2}=\langle Y(t), Y(t)\rangle+\left\langle Y^{\prime}(t), Y^{\prime}(t)\right\rangle .
$$

It follows from the Jacobi equation that

$$
\begin{aligned}
\left\{\langle Y, Y\rangle+\left\langle Y^{\prime}, Y^{\prime}\right\rangle\right\}^{\prime} & =2\left\{\left\langle Y, Y^{\prime}\right\rangle-\left\langle Y^{\prime}, R\left(Y, \dot{\gamma}_{v}\right) \dot{\gamma}_{v}\right\rangle\right\} \\
& \leq 2\left\{\|Y\|\left\|Y^{\prime}\right\|+K_{0}\left\|Y^{\prime}\right\|\|Y\|\left\|\dot{\gamma}_{v}\right\|^{2}\right\} \\
& =2\|Y\|\left\|Y^{\prime}\right\|\left\{1+K_{0}\|v\|^{2}\right\} \\
& \leq\left\{1+K_{0}\|v\|^{2}\right\}\left\{\langle Y, Y\rangle+\left\langle Y^{\prime}, Y^{\prime}\right\rangle\right\} .
\end{aligned}
$$

COROLlaRY 4.7. There is a constant $B>0$ such that

$$
\left.\frac{d}{d t}\left(\left\|d \varphi_{h_{\alpha}}^{t} \xi\right\|_{H_{\alpha}}\right)\right|_{t=0} \leq B\|\xi\|_{H_{\alpha}}
$$

whenever $\alpha \leq \alpha_{0}$ and $\xi \in T M_{\alpha}$.

Proof. This follows from Lemmas 4.6 and $4.1(\mathrm{a})$, since $\|v\|_{h_{\alpha}} \leq 2$ for any $v \in \mathcal{M}_{\alpha}$.

Proof of Proposition 4.3. Let $v_{0}=\dot{\beta}\left(-t_{0}(\alpha)\right)$. Then

$$
\left\{\varphi_{h_{\alpha}}^{t} v_{0}: 0 \leq t \leq t_{0}(\alpha)+t_{1}(\alpha)\right\}=\left\{\dot{\beta}(t):-t_{0}(\alpha) \leq t \leq t_{1}(\alpha)\right\}
$$

We see from (13) that $\dot{\beta}(t) \in \mathcal{M}_{\alpha}$ and $\operatorname{dist}_{H_{\alpha}}\left(\dot{\beta}(t), \partial M_{\alpha}\right) \geq \alpha>\varepsilon \alpha$ for $-t_{0}(\alpha) \leq t \leq t_{1}(\alpha)$. Choose $B$ as in Corollary 4.7 and $\varepsilon^{\prime}$ very small. It follows from Lemma 4.4 , Lemma 4.5 and (14) that, if $\tilde{g} \mid \mathcal{N}$ and $g \mid \mathcal{N}$ are sufficiently $C^{3}$ close and $\varepsilon^{\prime}$ is small enough, then

$$
\operatorname{dist}_{H_{\alpha}}\left(\varphi_{h_{\alpha}}^{\prime} v_{0}, \varphi_{h_{\alpha}}^{\prime} v_{0}\right) \leq \frac{\varepsilon^{\prime} \alpha}{B}\left\{\mathrm{e}^{B\left(t_{0}+t_{1}\right)}-1\right\} \leq \varepsilon \alpha
$$

for $0 \leq t \leq t_{0}(\alpha)+t_{1}(\alpha)$. It follows that

$$
\operatorname{dist}_{h_{\alpha}}(\beta(t), \tilde{\beta}(t)) \leq \varepsilon \alpha
$$

for $-t_{0}(\alpha) \leq t \leq t_{1}(\alpha)$. A similar argument, with time reversed, proves this inequality for $-t_{1}(\alpha) \leq t \leq-t_{0}(\alpha)$.

Now we make a comparison between the scalar Jacobi equations along $\beta$ and $\tilde{\beta}$,

$$
\begin{aligned}
& y^{\prime \prime}+K_{h_{\alpha}}(\beta(t)) y=0, \\
& y^{\prime \prime}+K_{\tilde{h}_{\alpha}}(\tilde{\beta}(t)) y=0 .
\end{aligned}
$$


We transform both equations by setting $\theta=\tan ^{-1}\left(y^{\prime} / y\right)$, to obtain

$$
\begin{aligned}
& \theta^{\prime}+\sin ^{2} \theta+K_{h_{\alpha}}(\beta(t)) \cos ^{2} \theta=0, \\
& \theta^{\prime}+\sin ^{2} \theta+K_{\tilde{h}_{\alpha}}(\tilde{\beta}(t)) \cos ^{2} \theta=0 .
\end{aligned}
$$

Note $\theta \in \mathbb{R} / \pi \mathbb{Z}$, which we identify with $(-\pi / 2, \pi / 2]$.

PROPOSITION 4.8. Let $\varepsilon>0$. Let $\theta$ and $\tilde{\theta}$ be the solutions of (17) and $\left(17^{\sim}\right)$ respectively with $\theta(0)=\theta_{0}=\tilde{\theta}(0)$. If $\tilde{\mathrm{g}} \mid \mathcal{N}$ and $\mathrm{g} \mid \mathcal{N}$ are close enough in the $C^{3}$ topology, then, for any $\alpha \leq \alpha_{0}$ and $\theta_{0} \in \mathbb{R} / \pi \mathbb{Z}$, we have $|\theta(t)-\tilde{\theta}(t)| \leq \varepsilon$ for $-t_{1}(\alpha) \leq t \leq t_{1}(\alpha)$.

Proof. We apply Lemma 4.4 with $X$ and $\tilde{X}$ as the vector fields on $\left[-t_{1}(\alpha), t_{1}(\alpha)\right] \times$ $\mathbb{R} / \pi \mathbb{Z}$ that define (17) and $\left(17^{2}\right)$ respectively. The linearization of $(17)$ is

$$
\Theta^{\prime}+2 \sin \theta \cos \theta\left\{1-K_{h_{\alpha}}(\beta(t))\right\} \Theta=0 .
$$

We see from this, (13) and Lemma 4.1(a) that $\Theta^{\prime} / \Theta$ is uniformly bounded for $\alpha \leq \alpha_{0}$ and $-t_{1}(\alpha) \leq t \leq t_{1}(\alpha)$. Thus the proposition will follow from Lemma 4.4 if we choose $\varepsilon^{\prime}>0$ small enough and show that

$$
\left|K_{h_{\alpha}}(\beta(t))-K_{\tilde{h}_{\alpha}}(\tilde{\beta}(t))\right| \leq \varepsilon^{\prime}
$$

whenever $\alpha \leq \alpha_{0}$ and $-t_{1}(\alpha) \leq \alpha \leq t_{1}(\alpha)$. We have

$$
\begin{aligned}
& \left|K_{h_{\alpha}}(\beta(t))+K_{\tilde{h}_{\alpha}}(\tilde{\beta}(t))\right| \\
& \quad \leq\left|K_{h_{\alpha}}(\beta(t))-K_{h_{\alpha}}(\tilde{\beta}(t))\right|+\left|K_{h_{\alpha}}(\tilde{\beta}(t))-K_{\tilde{h}_{\alpha}}(\tilde{\beta}(t))\right| .
\end{aligned}
$$

Proposition 4.3 and Lemma 4.1(b) show that the first term on the right hand side of (18) can be made less than $\frac{1}{2} \varepsilon^{\prime}$ if $\tilde{g} \mid \mathcal{N}$ and $g \mid \mathcal{N}$ are sufficiently $C^{3}$-close. Proposition 4.3 and (13) show that $\tilde{\beta}(t) \in \mathcal{N}_{\alpha}$. Hence, by Lemma 4.2, the second term on the right hand side of (18) is less than $\frac{1}{2} \varepsilon^{\prime}$ if $\tilde{g} \mid \mathcal{N}$ and $g \mid \mathcal{N}$ are sufficiently $C^{3}$-close.

Proposition 4.9. If $g \mid \mathcal{N}$ and $\tilde{g} \mid \mathcal{N}$ are close enough in the $C^{3}$ topology then, for any $\alpha \leq \alpha_{0}$ and any solution $y$ of $\left(16^{\tilde{)}}\right)$ with $y\left(-t_{1}(\alpha)\right) y^{\prime}\left(-t_{1}(\alpha)\right) \geq 0$, we have $y\left(t_{1}(\alpha)\right) y^{\prime}\left(t_{1}(\alpha)\right) \geq 0$.

Proof. The proposition is equivalent to the claim that if $\tilde{\theta}$ is a solution of $\left(17^{\sim}\right)$ with $0 \leq \tilde{\theta}\left(-t_{1}(\alpha)\right) \leq \pi / 2$, then $0 \leq \tilde{\theta}\left(t_{1}(\alpha)\right) \leq \pi / 2$. Let $\tilde{\theta}_{S}$ and $\tilde{\theta}_{C}$ be the solutions of (17) with $\tilde{\theta}_{S}(0)=\pi / 2$ and $\tilde{\theta}_{C}(0)=0$. Let $\theta_{S}$ and $\theta_{C}$ be the corresponding solutions of (17). Then $\alpha^{1 / 3} \tan \left[\theta_{S}\left(\alpha^{1 / 3} t\right)\right]=u_{S}(t)$ and $\alpha^{1 / 3} \tan \left[\theta_{C}\left(\alpha^{1 / 3} t\right)\right]=u_{C}(t)$, where $u_{S}$ and $u_{C}$ are the solutions of (5) defined in $\S 2$. It follows from Lemma 3.5 that there are constants $\theta_{1}$ and $\theta_{2}$ such that, for any $\alpha \leq \alpha_{0}$,

$$
0<\theta_{1} \leq \theta_{S}\left(t_{1}(\alpha)\right)<\theta_{C}\left(t_{1}(\alpha)\right) \leq \theta_{2}<\pi / 2
$$

Since $\theta_{S}$ and $\theta_{C}$ are odd functions, we also have

$$
0>-\theta_{1} \geq \theta_{S}\left(-t_{1}(\alpha)\right)>\theta_{C}\left(-t_{1}(\alpha)\right) \geq-\theta_{2}>-\pi / 2 .
$$

It follows from Proposition 4.8 that if $\tilde{g} \mid \mathcal{N}$ and $g \mid \mathcal{N}$ are close enough in the $C^{3}$ topology, there are constants $\tilde{\theta}_{1}$ and $\tilde{\theta}_{2}$ such that, for any $\alpha \leq \alpha_{0}$,

and

$$
0<\tilde{\theta}_{1} \leq \tilde{\theta}_{S}\left(t_{1}(\alpha)\right)<\tilde{\theta}_{C}\left(t_{1}(\alpha)\right) \leq \tilde{\theta}_{2}<\pi / 2
$$

$$
0>-\tilde{\theta}_{1} \geq \tilde{\theta}_{S}\left(-t_{1}(\alpha)\right)>\tilde{\theta}_{C}\left(-t_{1}(\alpha)\right) \geq-\tilde{\theta}_{2}>-\pi / 2 .
$$

This proves the proposition; see figure 2 . 


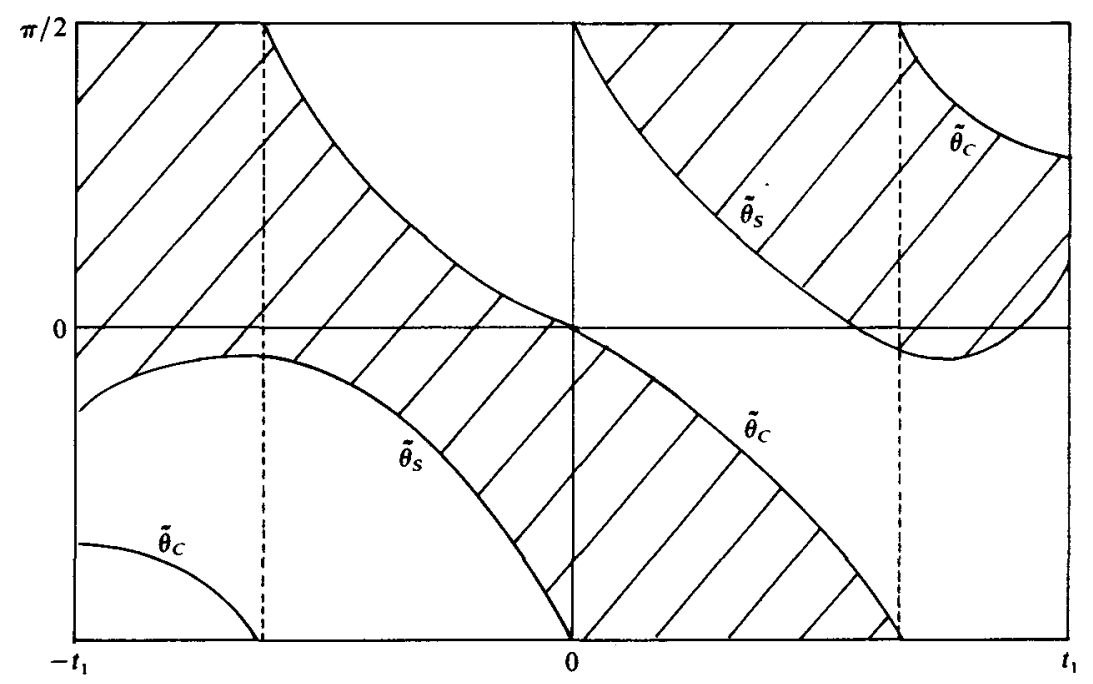

FIGURE 2

Proposition 4.10. Let $\mathscr{C}, \mathscr{D}$ and $\mathcal{N}$ be as described at the beginning of $\S 4$. If $g \mid \mathcal{N}$ and $\tilde{\mathrm{g}} \mid \mathcal{N}$ are close enough in the $C^{3}$ topology and $\tilde{\mathrm{g}}$ is close enough to $\mathrm{g}$ in the $C^{2}$ topology (throughout $\mathscr{D}$ ), then for any unit speed geodesic $\tilde{\gamma}$ in $(\mathscr{D}, \tilde{g})$ such that $\tilde{\gamma}(a) \in \partial \mathscr{D}$ and $\tilde{\gamma}(b) \in \partial \mathscr{D}$, and for any solution $\tilde{y}$ of the scalar Jacobi equation along $\tilde{\gamma}$,

$$
\tilde{y}^{\prime \prime}(t)+K_{\tilde{g}}(\tilde{\gamma}(t)) \tilde{y}(t)=0,
$$

with $\tilde{y} \tilde{y}^{\prime}(a) \geq 0$, we have $\tilde{y} \tilde{y}^{\prime}(b) \geq 0$.

Proof. As in the proof of Theorem 1.2 , we may assume that $(\mathscr{D}, \tilde{\boldsymbol{g}})$ has negative curvature outside $\mathscr{C}$ and consequently $\operatorname{dist}_{\tilde{\boldsymbol{g}}}(\mathscr{C}, \cdot)$ is a strictly convex function on $\mathscr{D} \backslash \mathscr{C}$. If $\tilde{y}$ does not enter $\mathscr{C}, \tilde{y} \tilde{y}^{\prime}(b) \geq 0$ follows from Lemma 1.5. Suppose that $\tilde{\gamma}$ enters $\mathscr{C}$, making angle $\alpha$ with $\partial \mathscr{C}$. We may assume that $a$ was chosen so that, as in the rest of $\S 4, \tilde{\gamma}\left(-T_{0}(\alpha)\right)$ is the point where $\tilde{\gamma}$ enters $\mathscr{C}$. Choose $\alpha_{0}$ as in Proposition 4.9. If $\alpha<\alpha_{0}$, it follows from Proposition 4.9 and Lemma 1.5 that $\tilde{y} \tilde{y}^{\prime}(b) \geq 0$. Now consider the case $\alpha \geq \alpha_{0}$. Clearly it is enough to prove that $\tilde{y} \tilde{y}^{\prime}(b) \geq 0$ for any solution of (19) with

$$
\left(\tilde{y}^{2}+\left(\tilde{y}^{\prime}\right)^{2}\right)\left(-T_{0}(\alpha)\right)=1 \text { and } \tilde{y} \tilde{y}^{\prime}\left(-T_{0}(\alpha)\right) \geq 0 .
$$

Choose $\delta$ such that $0<\delta<\operatorname{dist}_{g}(\partial \mathscr{C}, \partial \mathscr{D})$. As before let $\gamma$ be the geodesic of $(S, g)$ with $\dot{\gamma}\left(-T_{0}(\alpha)\right)=\dot{\gamma}\left(-T_{0}(\alpha)\right)$. Consider the scalar Jacobi equation along $\gamma$,

$$
\left.y^{\prime \prime}(t)+K_{g}(\gamma(t)) y(t)\right)=0 .
$$

It follows from Proposition 2.7 and Lemma 1.5 that, for any $\alpha$ with $\alpha_{0} \leq \alpha \leq \pi / 2$, any solution of (21) satisfying

$$
\left(y^{2}+\left(y^{\prime}\right)^{2}\right)\left(-T_{0}(\alpha)\right)=1 \text { and } y y^{\prime}\left(-T_{0}(\alpha)\right) \geq 0
$$

has $y y^{\prime}\left(T_{0}(\alpha)+\delta\right)>0$. An obvious compactness argument shows that there is $\varepsilon>0$ such that $y y^{\prime}\left(T_{0}(\alpha)+\delta\right)>\varepsilon$ whenever $\alpha_{0} \leq \alpha \leq \pi / 2$ and $y$ satisfies (21) and (22). Note that $T_{0}(\alpha)$ is uniformly bounded for $\alpha_{0} \leq \alpha \leq \pi / 2$. If $g$ and $\tilde{g}$ are close enough in the $C^{2}$ topology, then, for any geodesic $\tilde{\gamma}$ with $\alpha \geq \alpha_{0}$, the equations (19) and 
(21) will be close enough so that any solution $\tilde{y}$ of (19) satisfying (20) has $\tilde{y} \tilde{y}^{\prime}\left(T_{0}(\alpha)+\delta\right) \geq 0$. Moreover, we can assume that $\tilde{\gamma}$ and $\gamma$ are sufficiently close that $\tilde{\gamma}(t) \in \mathscr{D} \backslash \mathscr{C}$ for $T_{0}(\alpha)+\delta \leq t \leq b$. Then $\tilde{y} \tilde{y}^{\prime}(b) \geq 0$ by Lemma 1.5 .

This is the result that is needed to prove Theorem 1.2.

\section{Real analytic metrics}

To obtain real analytic metrics with Bernoulli geodesic flows we will need Theorem 5.1 below, which is based on H. Cartan's theorem B. We are grateful to S. Zucker for pointing out Cartan's theorem to us and explaining its application to our Lemma 5.2 .

THEOREM 5.1. Let $S$ be a compact real analytic surface with a $C^{\infty}$ Riemannian metric g. Suppose that $\gamma$ is a union of disjoint closed real analytic curves on $S$ and there exists a neighbourhood $\mathcal{N}$ of $\gamma$ on which $g$ is real analytic. Then for any positive integer $r$ there exists a real analytic Riemannian metric $\tilde{g}$ on $S$ such that $g$ and $\tilde{g}$ agree up to order $r$ on $\gamma$. Moreover $\tilde{g}$ can be taken arbitrarily close to $g$ in the $C^{\infty}$ topology.

Remark. It follows from Proposition 2.1 and the discussion after Theorem 1.1 that for any compact real analytic surface $S$ there exists a $C^{\infty}$ Riemannian metric $g$ and caps $\mathscr{C}_{1}, \ldots, \mathscr{C}_{n}$ satisfying the hypothesis of Theorem 1.2 such that each $\partial \mathscr{C}_{i}$ is a real analytic curve and there is a neighbourhood of $\bigcup \partial \mathscr{C}_{i}$ on which $g$ is real analytic. Thus Corollary 1.3 follows from Theorem 1.2 and Theorem 5.1.

LEMMA 5.2. Let $S$ be a real analytic surface and let $\gamma$ be a finite union of disjoint real analytic curves on $S$. Suppose that $f: S \rightarrow \mathbb{R}$ is $C^{\infty}$ and there is some neighbourhood $\mathcal{N}$ of $\gamma$ such that $f \mid \mathcal{N}$ is real analytic. Then given any positive integer $r$ there exists a real analytic function $\tilde{f} \mid S \rightarrow \mathbb{R}$ such that $f$ and $\tilde{f}$ agree up to order $r$ on $\gamma$.

Proof. Let $\mathcal{O}_{S}$ be the sheaf of real analytic functions on $S$ and let $\mathscr{G}$ be the subsheaf of real analytic functions which vanish up to order $r$ on $\gamma$. Then the short exact sequence

$$
0 \rightarrow \mathscr{G} \rightarrow \mathcal{O}_{S} \rightarrow \mathcal{O}_{S} / \mathscr{G} \rightarrow 0
$$

induces a long exact sequence

$$
0 \rightarrow H^{0}(S, \mathscr{G}) \rightarrow H^{0}\left(S, \mathcal{O}_{S}\right) \rightarrow H^{0}\left(S, \mathcal{O}_{S} / \mathscr{G}\right) \stackrel{\delta^{0}}{\rightarrow} H^{1}(S, \mathscr{G}) \rightarrow \cdots
$$

(See, e.g. [We].) Since the sheaf $\mathscr{G}$ is coherent, it follows from Theorem 3B of [C] that $H^{1}(S, \mathscr{G})=0$ and consequently the induced map $H^{0}\left(S, O_{S}\right) \rightarrow H^{0}\left(S, O_{S} / \mathscr{G}\right)$ is onto. Since the cohomology groups of degree 0 are the sections, the lemma follows.

Proof of Theorem 5.1. By [B], we may assume that $S$ is real analytically embedded in $\mathbb{R}^{m}$ so that $\gamma$ is real analytic and the metric $g \mid \mathcal{N}$ is real analytic. For $x=$ $\left(x_{1}, \ldots, x_{m}\right) \in S$, we define an inner product $\bar{g}$ on $T_{x} \mathbb{R}^{m}$ by $\bar{g} \mid T_{x} S=g$, and if $w \in T_{x} S$ and $v=\left(v_{1}, \ldots, v_{m}\right) \in\left(T_{x} S\right)^{\perp}$ then $\bar{g}(v, v)=v_{1}^{2}+\cdots+v_{m}^{2}$ and $\bar{g}(v, w)=0$. Define $\bar{g}_{i j}$ : $S \rightarrow \mathbb{R}, 1 \leq i, j \leq m$, by

$$
\overline{\boldsymbol{g}}_{i j}(x)=\overline{\boldsymbol{g}}_{x}\left(\frac{\partial}{\partial x_{i}}, \frac{\partial}{\partial x_{j}}\right)
$$


Clearly $\left(\bar{g}_{i j}(x)\right)_{1 \leq i, j \leq m}$ is a positive definite matrix for all $x \in S$. Also, for $1 \leq i, j \leq m$, $\overline{\boldsymbol{g}}_{i j}$ is $C^{\infty}$ and $\overline{\boldsymbol{g}}_{i j} \mid \mathcal{N}$ is real analytic. By Proposition 15 of [C] there exists a real analytic function $F$ whose zero set is $\gamma$. By replacing $F$ by one of its powers, if necessary, we may assume that $F$ vanishes up to order $r$ on $\gamma$. By the inequality of Lojasiewicz (Theorem 4.1 in Chapter IV of [Ma1] there exist constants $c, r_{1}>0$ such that for $x \in S$,

$$
|F(x)| \geq c(\operatorname{dist}(x, \gamma))^{r_{1}} \text {. }
$$

Now consider the quotient $Q=f / F$, where $f: S \rightarrow \mathbb{R}^{2}$ is a $C^{\infty}$ function which vanishes to some order $r_{2}$ on $\gamma$. It follows by successive implicit differentiation of $f=F Q$ that if $r_{2}=r_{2}\left(k, r_{1}\right)$ is sufficiently large and we set $Q=0$ on $\gamma$, then $Q$ is $C^{k}$ on $S$. From Lemma 5.2 we obtain real analytic functions $h_{i j}$ such that $\bar{g}_{i j}$ and $h_{i j}$ agree up to order $r_{2}$ on $\gamma$. Then $\left(\bar{g}_{i j}-h_{i j}\right) / F$ is $C^{k}$ on $S$ (if we define this quotient to be zero on $\gamma$ ) and there are real analytic functions $\beta_{i j}$ such that

$$
\frac{\bar{g}_{i j}-h_{i j}}{F}-\beta_{i j}
$$

is close to 0 in the $C^{k}$ topology. Since the $\beta_{i j}$ 's are chosen after $F$, we can get $\bar{g}_{i j}-\left(h_{i j}+\beta_{i j} F\right)$ sufficiently close to 0 in the $C^{k}$ topology so that

$$
\left(h_{i j}(x)+\beta_{i j}(x) F(x)\right)_{1 \leq i, j \leq m}
$$

is positive definite for all $x \in S$ and the metric $\tilde{g}$ on $S$ obtained by restricting the inner product obtained from $\left(h_{i j}+\beta_{i j} F\right)_{1 \leq i, j \leq m}$ to $T S$ is $C^{k}$ close to $g$. Also $\tilde{g}$ is real analytic and agrees with $g$ up to order $r$ on $\gamma$.

\section{REFERENCES}

[BBB] W. Ballmann, M. Brin \& K. Burns. On surfaces with no conjugate points. J. Diff. Geom. 25 (1987), 249-273.

[BGS] W. Ballmann, M. Gromov \& V. Schroeder. Manifolds of Nonpositive Curvature. Progress in Mathematics 61, Birkhäuser: 1985.

[B] S. Bochner. Analytic mapping of compact Riemann spaces into Euclidean space. Duke Math. J. 3 (1937), 339-354.

[Bu] L. A. Bunimovich. Personal communication.

[BG] K. Burns \& M. Gerber. Continuous invariant cone families and ergodicity of flows in dimension three. Preprint. 1987, To appear in Ergod. Th. \& Dynam. Sys.

[C] H. Cartan. Variétés analytiques réelles et variétés analytiques complexes. Bull. Soc. Math. France 85 (1957), 77-99.

[CE] J. Cheeger \& D. Ebin. Comparison Theorems in Riemannian Geometry. North-Holland: Amsterdam, 1975.

[D1] V. J. Donnay. Geodesic flow on $S^{2}$ with positive measure entropy. Preprint, 1986.

[D2] V. J. Donnay. Geodesic flow on the two sphere with positive entropy. Ph.D. Thesis, New York University, 1986.

[D3] V. J. Donnay. Geodesic flow on the two-sphere, Part I: Positive measure entropy. Preprint, 1987.

[D4] V. J. Donnay. Geodesic flow on the two-sphere, Part II: Ergodicity. Preprint, 1987.

[FLP] A. Fathi, F. Laudenbach \& V. Poénaru. Travaux de Thurston sur les Surfaces. Astérisque 66-67, Soc. Math. France: Paris, 1979.

[Ka] A. Katok. Invariant cone families and stochastic properties of smooth dynamical systems. Preprint, 1987. 
[K1] W. Klingenberg. Riemannian Geometry. De Gruyter Studies in Mathematics, 1982.

[Ma1] B. Malgrange. Ideals of Differentiable Functions. Oxford University Press, 1966.

[M] A. Manning. More topological entropy for geodesic flows. Lecture Notes in Mathematics, vol. 898, pp. 243-249. Springer: New York, 1980.

[O] R. Osserman. Talk at Cal. Tech., 1985.

[We] R. O. Wells. Differential Analysis on Complex Manifolds. Springer: New York, 1980.

[W] M. Wojtkowski. Invariant families of cones and Lyapunov exponents. Ergod. Th. \& Dynam. Sys. 5 (1985), 145-161. 